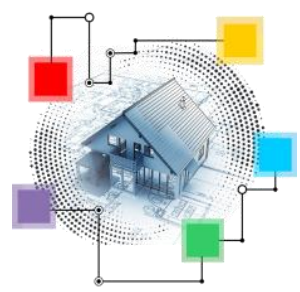

\author{
XIII SIMPÓSIO NACIONAL DE SISTEMAS PREDIAIS \\ DESEMPENHO E INOVAÇÃO \\ DE SISTEMAS PREDIAIS HIDRÁULICOS \\ SÃO PAULO - 04 DE OUTURO DE 2019
}

\title{
Avaliação da produção de água não potável descentralizada e a produção de água potável centralizada na RMSP1
}

\section{Evaluation of decentralized non-potable water production and centralized water production in SPMR}

\author{
PATUCCI, Renato Augusto'; OLIVEIRA, Lúcia Helena2; \\ KUROKAWA, Fernando Akira ${ }^{3}$
}

\author{
${ }^{1}$ Escola Politécnica da USP, Av. Prof. Almeida Prado, Travessa 2 no 83 - Edifício da Engenharia \\ Civil, renato.patucci@usp.br \\ ${ }^{2}$ Escola Politécnica da USP, lucia.helena@usp.br \\ ${ }^{3}$ Escola Politécnica da USP, fernando.kurokawa@usp.br
}

\begin{abstract}
RESUMO
A população no Brasil atingirá seu pico aproximadamente no ano 2050, período em que a demanda por recursos naturais crescerá, sobretudo a da água. $\mathrm{O}$ adensamento populacional reduz a disponibilidade hídrica por habitante, além de prejudicar a qualidade dos mananciais. O objetivo desse artigo é aplicar um modelo matemático para avaliar se a utilização de sistemas prediais de água não potável podem ser uma fonte alternativa economicamente viável, em relação à ampliação do sistema centralizado de produção de água potável. A água não potável pode atender uma parte da demanda dos usos domésticos numa proporção estimada de $80 \%$ de consumo de água potável e $20 \%$ de consumo em fins que não demandam água potável. Realizou-se pesquisa bibliográfica e foram coletados custos de execução, operação e manutenção, de um sistema predial de água não potável recuperada (SPANP-R) descentralizado individual e do sistema produtor São Lourenço (SPSL). O modelo matemático foi formulado e aplicado aos dados coletados, que foram ajustados para levar em consideração o ganho decorrente da utilização em larga escala dos sistemas de água não potável. Os resultados indicam que a produção de água não potável em edifícios tem menor viabilidade econômica em relação ao SPSL no cenário avaliado.
\end{abstract}

Palavras-chave: Sistema predial de água não potável, Sistema centralizado de água potável, Tomada de decisão.

\begin{abstract}
The population in Brazil will reach its peak approximately in the year 2050, until then, the demand for natural resources will grow, especially water. Population densification reduces per capita water availability, as well as impairing the quality of water sources. The aim of this article is to apply a mathematical model to evaluate if the use of non-potable water systems can be an economically viable alternative source in relation to the expansion of the centralized drinking water system. Non-potable water can meet a portion of the demand for domestic uses, the approximate proportion of $80 \%$ of drinking water consumption and $20 \%$ of consumption can be considered for purposes that do not require potable water. Bibliographic review was carried out and costs were collected for the construction, operation and maintenance of a decentralized individual non-potable water production system and the São Lourenço production system. The mathematical model was formulated and applied under the collected data, which were adjusted to take into account the gain resulting from the large-scale use of non-potable water systems. The results indicate that the production of non-potable water in buildings has less economic viability compared to SPSL in the evaluated scenario.
\end{abstract}

Keywords: Non-potable water system, Centralized drinking water system, Decision making.

\footnotetext{
${ }^{1}$ PATUCCI, R. A.; OLIVEIRA, L. H.; KUROKAWA, F. A. Avaliação da produção de água não potável descentralizada e a produção de água potável centralizada na RMSP. In: SIMPÓSIO NACIONAL DE SISTEMAS PREDIAIS DESEMPENHO E INOVAÇÃO DE SISTMAS PREDIAIS HIDRAULICOS, 13., 2019, São Paulo. Anais... São Paulo: SISPRED, 2019.
} 


\section{INTRODUÇÃO}

A população no Brasil continuará a crescer até atingir o pico por volta do ano de 2050 (ONU, 2015) e na macrometrópole de São Paulo o crescimento será de aproximadamente 9\% no período de 2018 a 2035 ANA (2007). Esse crescimento populacional ocorrerá com a ampliação da urbanização, que exerce forte pressão no aumento do consumo de água.

Fontes alternativas geram novos recursos de água e a adoção das mesmas possibilita que ampliações do sistema de abastecimento centralizado sejam evitadas ou prorrogadas (DAIGER,2009). Durante os anos de 2015 a 2018, o Sistema Produtor São Lourenço (SPSL) foi construído para abastecimento da Região Metropolitana de São Paulo.

O objetivo desse artigo é aplicar um modelo matemático de tomada de decisão com base na Programação Inteira para avaliar a utilização de sistemas prediais de água não potável recuperada (SPANP-R) em larga escala, tendo em vista a possibilidade de ser uma fonte alternativa de água economicamente viável, em relação à implantação do SPSL.

\section{FONTES DE ÁGUA PARA ABASTECIMENTO URBANO}

A demanda crescente de água potável é majoritariamente atendida pelo sistema centralizado de abastecimento, em que se verificam problemas de escassez e de ordem econômica, devido às distâncias, cada vez maiores, dos mananciais, bem como, à necessidade de tratamentos mais intensos causados pela poluição da água (OLIVEIRA e GONÇALVES, 1999).

Entretanto, a água não potável pode atender uma parte da demanda dos usos domésticos que prescindam de água potável. Como parâmetro médio, pode-se considerar a proporção aproximada de $80 \%$ de consumo de água potável e $20 \%$ de consumo em fins que não demandam água potável (Patucci et al., 2018).

\subsection{Sistema de produção de água potável}

A ampliação da oferta de água potável que abastece a RMSP, entre os anos de 2015 a 2018, se deu através da construção do SPSL, que realiza a captação de água no Rio Juquiá com uma vazão média de $4,7 \mathrm{~m} 3 / \mathrm{s}$. A captação ocorre na cota $645 \mathrm{~m}$ acima do nível do mar, e exige a elevação do fluxo em $3.900 \mathrm{kPa}$ devido o elevado desnível topográfico da Serra do Paranapiacaba.

A perda de água, entre produção e o consumo final no sistema interligado metropolitano (SIM) é de 33\%, e o consumo médio diário per capita no SIM foi de 138,4 litros (SNIS, 2015). Dessa forma, é previsto que o SPSL atenda uma população de 1.996.131 habitantes.

\subsection{Sistema de produção de água não potável}

Conforme Marques e Oliveira (2014), as águas cinzas são aquelas advindas de aparelhos sanitários, excluindo os efluentes de bacias sanitárias, em alguns casos, excluem-se também o efluente da pia de cozinha e da máquina de lavar louças.

Essa fonte apresenta a importante característica, de ter o seu perfil de produção proporcional à demanda de água não potável. Esse atributo, característico das águas residuárias, é fundamental para a avaliação da viabilidade técnica e econômica dessa fonte.

Os sistemas descentralizados individuais atendem a um único edifício e sistemas descentralizados em grupo atendem a um conjunto de edifícios (OLIVEIRA et al., 2014). 
Neste trabalho, coletaram-se dados de custos de construção, manutenção e operação do SPANP-R de um edifício de cinquenta e seis apartamentos no bairro da Saúde, na cidade São Paulo.

Para a avaliação do ganho de eficiência com o aumento da escala de diferentes concepções de sistemas, foi realizada a simulação dos custos de implantação, manutenção e operação do SPANP-R para atender dois e dez edifícios, com cento e doze e quinhentos e sessenta unidades no total, representando um sistema descentralizado em grupo e centralizado, respectivamente.

\section{MATERIAIS E MÉTODOS}

Para o alcance dos objetivos foi utilizado um modelo matemático para a tomada de decisão com base na PI, desenvolvido por Dias (2017). A solução do modelo foi obtida com o auxílio do software LINDO ${ }^{\mathrm{TM}}$. O estudo iniciou-se por meio de pesquisa bibliográfica, para avaliar as experiências de implantação de SPANP-R em diferentes localidades. Também foram verificadas as formas de projetar as economias de escala, relacionadas à implantação em larga escala dos SPANP-R, para alcançar o porte da população atendida pelo SPSL.

\subsection{Coleta de dados}

Foram coletados dados relativos aos custos de implantação, operação e manutenção do SPSL, através principalmente do contrato público de concessão administrativa, cujo prazo é de 25 anos, sendo aproximadamente 5 anos de construção e 20 anos de operação.

Considerou-se também os valores do contrato específico para o gerenciamento das obras desse empreendimento e os custos para ampliação da capacidade de tratamento de esgoto, decorrentes do acréscimo de efluentes a serem gerados com o funcionamento do SPSL.

Os mesmos dados foram obtidos de um SPANP-R, que utiliza a tecnologia de disco biológico rotativo, instalado em um edifício residencial na RMSP. Foram coletados os custos de implantação do sistema diretamente com a construtora. Os dados dos custos de operação e de manutenção do SPANP-R foram obtidos por meio de visita ao edifício e contato com o síndico.

\subsection{Ajuste de parâmetros do modelo e a população atendida em cada sistema}

Foi realizado um ajuste de parâmetros do modelo, para projetar os dados de custos de construção, operação e manutenção obtidos, para um sistema com dois edifícios compartilhando o sistema de tratamento, representando um sistema descentralizado em grupo, outro sistema com dez edifícios representando um sistema centralizado de produção de água não potável.

A população do edifício com SPANP-R descentralizado individual é de 224 pessoas, o condomínio com SPANP-R descentralizado em grupo abriga 448 pessoas, e o condomínio com SPANP-R centralizado possui 2.240 pessoas. Dessa forma, como a parcela de água que pode ser substituída por água não potável em residências representa cerca de $20 \%$ do total consumido, a população equivalente atendida por cada sistema encontra-se apresentada na Tabela 1. 
TABELA 1 - População equivalente atendida pelos sistemas

\begin{tabular}{c|c|l|c}
\hline & $\mathbf{P}$ & Tipo de sistema $(\boldsymbol{i})$ & População equivalente atendida (pessoas) \\
\hline \multirow{2}{*}{$\boldsymbol{z}$} & $\mathrm{P}_{1}$ & Descentralizado individual & 45 \\
\cline { 2 - 4 } & $\mathrm{P}_{2}$ & Descentralizado em grupo & 90 \\
\cline { 2 - 4 } & $\mathrm{P}_{3}$ & Centralizado & 448 \\
\hline \multirow{2}{*}{} & $\mathrm{P}_{4}$ & SPSL & 1.966 .131 \\
\hline
\end{tabular}

Fonte: Os autores (2019).

\subsection{Modelo de Programação Inteira}

Foi aplicado um modelo matemático para tomada de decisão, com base no problema de Programação Inteira (PI), desenvolvido por Dias (2017b). O modelo verifica a combinação que promove o menor custo total acumulado em 20 anos de operação dos sistemas em diferentes cenários. A formulação é dada pela Equação (1).

$$
\begin{gathered}
(M I N) Z=A_{11} X_{11}+A_{12} X_{12}+A_{13} X_{13}+A_{21} X_{21}+A_{22} X_{22}+A_{23} X_{23}+A_{31} X_{31} \\
+A_{32} X_{32}+A_{33} X_{33}+A_{41} X_{41}+A_{42} X_{42}+A_{43} X_{43} \\
X_{12}-X_{11} \leq 0 \\
X_{13}-X_{12} \leq 0 \\
X_{22}-X_{21} \leq 0 \\
X_{23}-X_{22} \leq 0 \\
X_{32}-X_{31} \leq 0 \\
X_{33}-X_{32} \leq 0 \\
X_{42}-X_{41} \leq 0 \\
X_{43}-X_{42} \leq 0 \\
\text { s.a. }\left\{\begin{array}{c}
\text { (eq. }) \\
P_{1} X_{11}+P_{2} X_{21}+P_{3} X_{31}+P_{4} X_{41} \geq \text { População } o_{\text {min }} \\
P_{1} X_{12}+P_{2} X_{22}+P_{3} X_{32}+P_{4} X_{42} \geq \text { População } o_{\text {min }} \\
P_{1} X_{13}+P_{2} X_{23}+P_{3} X_{33}+P_{4} X_{43} \geq \text { População } o_{\text {min }} \\
X_{i j} \in \mathbb{Z}_{+}
\end{array}\right.
\end{gathered}
$$

Em que $X_{i j}$ representa a implantação ou não de determinado sistema, i corresponde ao tipo de sistema ( $\mathrm{i}=1,2,3$ e 4$)$ e $\mathrm{j}$ o tipo de custo associado $(\mathrm{j}=1,2$, e 3$)$ e os coeficientes $A_{i 1}, A_{i 2}$ e $A_{i 3}$ correspondem aos custos de implantação, manutenção e operação, respectivamente, de um determinado tipo de tratamento do sistema. A variável $P_{i}$ representa a população equivalente atendida pelo tipo de sistema $i$. Os custos acumulados de manutenção e operação durante a vida útil dos sistemas são calculados de acordo com as Equações (2) e (3), respectivamente.

$$
\begin{aligned}
A_{i 2} & =\sum_{i=1}^{n} M_{i} \times E E_{i} \times(1+1 \%)^{n} \\
A_{i 3} & =\sum_{i=1}^{n} O_{i} \times E E_{i} \times(1+3 \%)^{n}
\end{aligned}
$$


Em que:

- $M_{i}$ é o custo de manutenção do sistema tipo $i$ no primeiro ano de operação;

- $O_{i}$ é o custo de operação do sistema tipo $i$ no primeiro ano de operação;

- $E E_{i}$ corresponde ao efeito escala no sistema tipo $i$;

- $n$ caracteriza o ano de funcionamento do sistema escolhido para a análise;

De acordo com Dias (2017), a taxa de aumento anual do custo de manutenção de cada sistema pode ser considerada como $1 \%$ a.a., e a taxa de aumento anual do custo de operação foi considerada como o crescimento médio anual, acima da inflação, da energia elétrica, que foi de 3\% a.a. de acordo com o histórico dos últimos 15 anos (ANEEL, 2003; ANEEL, 2018 e Portal Brasil, 2018).

A solução do problema foi obtida através da utilização do software LINDO ${ }^{\mathrm{TM}}$.

\section{APLICAÇÃO DO MODELO - RESULTADOS}

Conforme apresentado na Tabela 1, para haver produção equivalente da demanda de 1.966.131 pessoas, seriam necessários 43.887 edifícios com SPANP-R descentralizado individual, 21.944 descentralizados em grupo ou 4.389 centralizados.

Guo et al. (2014) avaliaram o impacto de redução de custos devido ao efeito escala em sistemas reúso. Com base nesse estudo e aplicando coeficientes das economias de escala sobre os dados brutos coletados, obtêm-se os custos de construção, operação e manutenção, durante a vida útil dos sistemas, conforme apresentado na Tabela 2.

TABELA 2 - Custos de construção, operação e manutenção acumulados - cenário com efeito escala

\begin{tabular}{l|c|c|c|c|c|c|c|c|c}
\hline \multirow{2}{*}{ Sistema } & \multirow{2}{*}{$\begin{array}{c}\text { Construção } \\
(\mathbf{U S \$ ~ 1 . 0 0 0 )}\end{array}$} & \multicolumn{2}{|c|}{$\mathbf{5}$ anos } & \multicolumn{2}{c|}{$\mathbf{1 0}$ anos } & \multicolumn{2}{c|}{$\mathbf{1 5}$ anos } & \multicolumn{2}{c}{$\mathbf{2 0}$ anos } \\
\cline { 3 - 9 } & $\mathbf{O}$ & $\mathbf{M}$ & $\mathbf{O}$ & $\mathbf{M}$ & $\mathbf{O}$ & $\mathbf{M}$ & $\mathbf{O}$ & $\mathbf{M}$ \\
\hline Desc. ind. & 59,7 & 19,8 & 2,2 & 39,5 & 4,4 & 59,3 & 6,6 & 79,1 & 8,8 \\
\hline Desc. gru. & 109,0 & 34,0 & 4,3 & 68,0 & 8,6 & 102,0 & 12,9 & 135,9 & 17,1 \\
\hline Centrali. & 475,6 & 127,8 & 18,0 & 255,7 & 36,0 & 383,5 & 53,9 & 511,4 & 71,9 \\
\hline SPSL & 1.248 .914 & 268.296 & 24.898 & 531.591 & 49.797 & 804.887 & 74.695 & 1.073 .182 & 99.593 \\
\hline
\end{tabular}

O - Operação

Fonte: Os autores (2019).

M - Manutenção

Desc. ind. - descentralizado individual

Desc. gru. - descentralizado em grupo

Centrali. - centralizado

Esses dados indicam que os custos de operação e de manutenção ao final de 20 anos, são superiores aos custos de implantação nos quatro sistemas. Isso mostra que, no longo prazo, tanto nos sistemas de tratamento que atendem pequenas populações, quanto no sistema centralizado de abastecimento de água potável, os custos de operação e de manutenção tendem a ser mais importantes do que os custos de implantação.

Por meio da aplicação dos coeficientes da Tabela 2 na Equação 1 e com o emprego do software LINDO ${ }^{\mathrm{TM}}$, obteve-se os resultados apresentados na Figura 1. 
FIGURA 1 - Custo total acumulado durante os 20 primeiros anos de operação

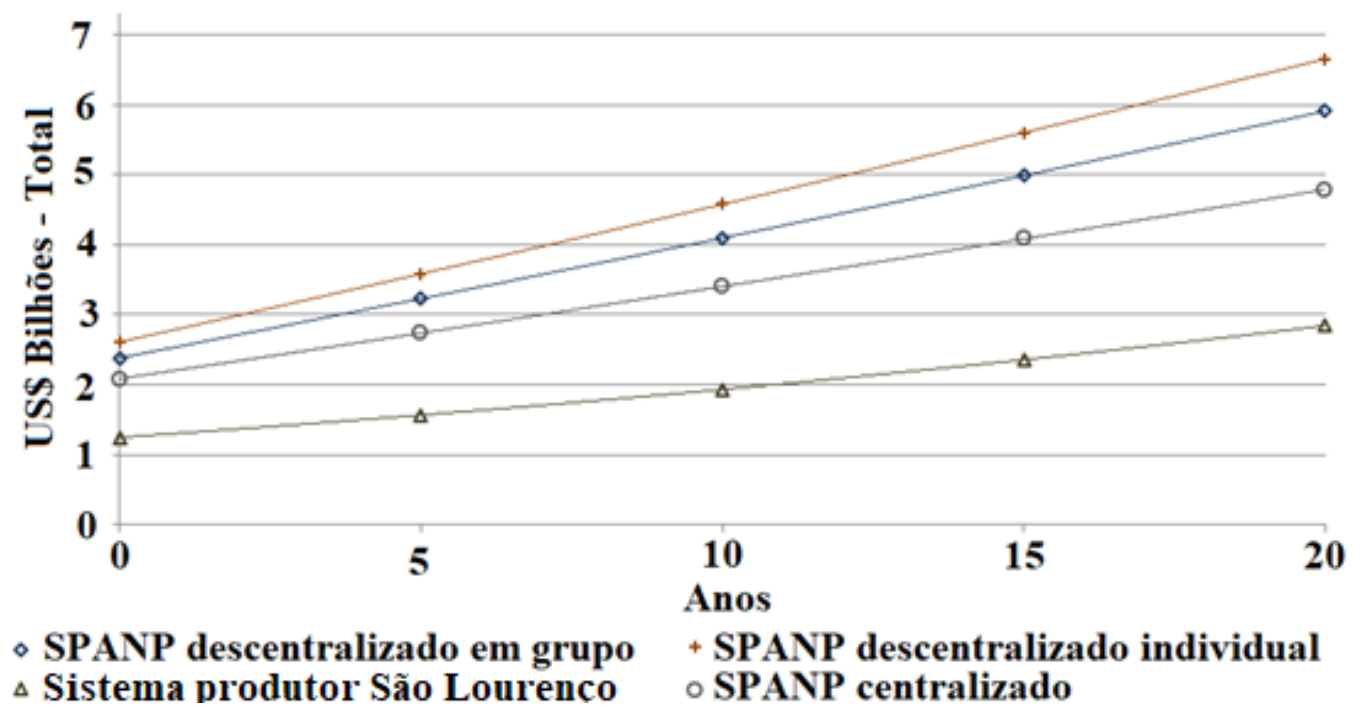

Fonte: Os autores (2019).

Na Figura 1, observa-se que o impacto do aumento dos custos da tarifa de energia elétrica, é muito relevante, e mais significativo no SPSL. Os SPANP-R apresentam custos operacionais mais previsíveis, uma vez que o principal custo é relacionado à terceirização do serviço de monitoramento do sistema, que é realizado por meio de um contrato que tem regras de reajustamento definidas.

\section{CONCLUSÕES}

Com base nos princípios da Programação Inteira, foi possível aplicar um modelo para tomada de decisão, apresentando qual alternativa fornece o menor custo total acumulado durante 20 anos de operação dos sistemas.

A verificação de uma tecnologia incipiente de tratamento de efluentes de pequena escala, quando avaliada para a aplicação em larga escala, necessita de extrapolação quanto os custos para capturar o benefício do efeito escala. Essa extrapolação deve ser realizada criteriosamente, tanto para não subdimensionar os ganhos possíveis, quanto para não ocorrer o superdimensionamento.

Os resultados indicam que a produção de água não potável em edifícios, através da tecnologia de disco biológico rotativo, tem menor viabilidade econômica em relação ao Sistema Produtor São Lourenço no cenário avaliado.

No entanto, existem outras tecnologias para tratamento de fontes alternativas não avaliadas. E há a tendência de aumento de custos, de um futuro sistema de produção de água potável de grande porte, em relação ao Sistema Produtor São Lourenço. Dessa forma, torna-se importante a consideração dessas variáveis para futuras avaliações.

\section{REFERÊNCIAS}

AGÊNCIA NACIONAL DE ENERGIA ELÉTRICA (ANEEL). Tarifa de energia ELETROPAULO - 2003. Disponível em: <http://www.energetica.eng.br/wa_files/ Tarifas_202003_20-_202005.pdf $>$. Acesso em: 20 de outubro de 2018. 
AGÊNCIA NACIONAL DE ENERGIA ELÉTRICA (ANEEL). Tarifa de energia ELETROPAULO - 2018. Disponível em: <http://www2.aneel.gov.br/cedoc/ reh20182412ti.pdf>. Acesso em: 20 de outubro de 2018e.

AGÊNCIA NACIONAL DE ÁGUAS (ANA). Disponibilidade e Demandas de Recursos Hídricos no Brasil - Caderno de Recursos Hídricos 2. Brasília, 2007.

DAIGER, G.T. Evolving Urban Water and Residuals Management Paradigms: Water Reclamation and Reuse, Decentralization and Resource Recovery. Water Environment Research, v. 81:8, p. 809-823, 2009.

DIAS C.M., Modelos para a tomada de decisão quanto ao tipo de sistema predial de água não potável. 2017. 221 p. Dissertação - Escola Politécnica da Universidade de São Paulo, São Paulo, 28 de abril de 2017.

DIAS, C. M.; OLIVEIRA, L. H.; KOROKAWA, F. A. A mathematical model for decisionmaking of a non-potable water system in residential buildings: decentralized in clusters or individual decentralized? International Symposium of CIB W062, v.1, p.283-293, 2017b.

GUO, T; ENGLEHARDT, J.D.; WU, T. Review of cost versus scale: water and wastewater treatment and reuse processes. Water Science \& Technology. v. 69, p. 223-234. 2014.

MARQUES, I. G.; OLIVEIRA L. H., Padronização de terminologia e de conceitos de sistemas prediais de água não potável. Conselho Brasileiro de Construção Sustentável (CBCS). São Paulo, 2014. 9 p.

OLIVEIRA, L. H., GONÇALVES O. M., Metodologia para implantação de programa de uso racional da água em edifícios. 1a ed. São Paulo; EPUSP-PCC, 1999. 18 p.

OLIVEIRA, L. H.; CORRÊA F. R.; SANTOS, M. O. Sistemas de água não potável: centralizados ou descentralizados? Revista Hydro, v. 88, p. 54-57, 2014.

ORGANIZAÇÃO DAS NAÇÕES UNIDAS (ONU). Novo estudo da ONU indica que mundo terá 11 bilhões de habitantes em 2100. Disponível em: <www.nacoesunidas.org/novo-estudo-daonu-indica-que-mundo-tera-11-bilhoes-de-habitantes-em-2100/〉. Acesso em: 05 de setembro de 2016.

PATUCCI, R. P.; OLIVEIRA, L. H.; KUROKAWA, F. A.; MIELLI, V. G. Tomada de decisão entre a produção de água não potável em edifícios residenciais e água potável no Sistema Produtor São Lourenço. In: Encontro Nacional de Tecnologia do Ambiente Construído, 18., 2018, São Paulo. Anais... Foz do Iguaçu: ENTAC, 2018.

PORTAL BRASIL. Índice Geral de Preços do Mercado. Disponível em: <https://www.portalbrasil.net/igpm.htm>. Acesso em: 6 de junho de 2018.

SAUTCHÚK, C. A. et al. Conservação e reuso da água em edificações. São Paulo, 2005, 152 p. Disponível em: <http://www.fiesp.com.br/indices-pesquisas-e-publicacoes/conservacao-ereuso-de-aguas-em-edificacoes-2005>. Acesso em 25 de agosto de 2017.

SISTEMA NACIONAL DE INFORMAÇÕES SOBRE SANEAMENTO (SNIS). Diagnóstico dos serviços de água e esgotos - 2015. Disponível em: <http://www.snis.gov.br/diagnostico-agua-eesgotos/diagnostico-ae-2015>. Acesso em: 2 nov. 2017. 